\title{
Postoperative Pain Management After Sternotomy In Off-Pump Coronary Artery Bypass Graft (CABG) Surgery - A Comparative Study Between NSAID (Diclofenac Sodium) and Opioid (Pethidine)
}

\author{
Kamrul Hasan ${ }^{1}$, Zerzina Rahman², Ayesha Sultana ${ }^{1}$, Najib Ahsan ${ }^{3}$ \\ ${ }^{1}$ Consultant ${ }^{2}$ Associate Professor, ${ }^{3}$ Medical Officer, Dept. of Anesthesia, Analgesia \& Intensive Care Medicine (Cardiac Anesthesia Wing), \\ BSMMU, Dhaka.
}

\begin{abstract}
:
Background: Traditionally, postoperative pain has been managed either reactively with drugs given as needed or proactively with continuous infusion of analgesics. Objectives:The present prospective comparative study was carried out to find difference in efficacy between opioid and NSAID (Non-stroid anti-inflammatory drugs) in the post-sternotomy pain management following off pump coronary bypass graft surgery. Methods: A total of 30 patients were randomly divided into two groups. - 15 patients were treated with NSAID (Diclofenac sodium) and 15 patients with opioid (pethidine) which are not commonly used in cardiac surgery. Patients ranging from 40 - 60 years with ASA Grade I \& II who underwent off-pump CABG with median sternotomy were included in the study. Statistics: The test statistics used to analyze the data were Chi-square Test and repeated measure ANOVA. Result \& conclusion: The study concluded that the intensity of post-sternotomy pain was inappreciably higher in the NSAID group than that in the opioid group throughout the whole period of observation suggesting that opioid (pethidine) would be a promising analgesic in the post-sternotomy pain management than NSAID (diclofenac sodium) $(\mathrm{p}=0.045)$.
\end{abstract}

Key words: Post-sternotomy pain: OPCAB: choice of analgesic.

[BSMMU J 2010; 3(2): 91-96]

\section{Introduction:}

Coronary heart disease is a life threatening condition for human population. During surgical intervention like Coronary Artery Bypass Graft (CABG), median sternotomy is the best approach for a clear visualization of heart \& associated viscera. Median sternotomy is a type of surgical procedure in which a midline vertical incision is made along the sternum after which the sternum itself is divided or cracked. ${ }^{1}$ Cardiac procedure requiring median sternotomy includes coronary artery bypass grafting, valve replacement, repair of a variety of congenital cardiac diseases. Post-sternotomy pain is mostly musculoskeletal \& myofascial. These painful conditions are jointly called post sternotomy pain syndrome ${ }^{2}$. Per-operatively it is controlled by large bolus intravenous infusion of opioid viz morphine or fentanyl. Thoracotomy, sternotomy and the placement of pleural chest tubes results in considerable pain in the post operative period in patients undergoing $\mathrm{CABG}^{3}$. Traditionally opioids and NSAID are used for post operative pain management after sternotomy.

Address for Correspondence: Dr. Zerzina Rahman, Associate Professor; Department of Anesthesia, Analgesia \& Intensive Care Medicine (Cardiac Anesthesia wing); Bangabandhu Sheikh Mujib Medical University, Dhaka, Bangladesh. E-mail: bannya84@gmail.com
Inadequate analgesia causes respiratory, haemodynamic, endocrine and metabolic complications. The preferred drugs for postoperative pain management are opioids. Pethidine is not a common opioid chosen for pain management after cardiac surgery though it is used as a common post-operative analgesic after general surgery. Usually morphine is used for haemodynamic stability after open heart surgery but it has a series of side-effects like nausea, vomiting, constipation, respiratory depression. NSAIDs are also used for analgesia. It also has some-side effects such as gastro-intestinal disturbance, renal impairment, decreased platelet function, impaired coagulation etc ${ }^{4}$. In this study we assessed and compared the efficacy of two different analgesics to relieve post sternotomy pain with an assumption that opioid (Pethidine) may have better effects than NSAID (Diclofenac Sodium) in controlling the post-operative pain following sternotomy for OPCAB (Off Pump Coronary artery bypass) surgery

Pain is more than just a physical process; it is a complex, subjective phenomenon ${ }^{5}$. Pain can impair the haematologic, immune, hormonal, cardiac, and respiratory systems. ${ }^{6}$ Pain also can limit mobility ${ }^{7}$, interfere with sleep and rest, and contribute to agitation, Psychosis, aggressive behavior, and delirium ${ }^{8}$. 
Surgical centers need to pay attention to pain management, because there appears to be a direct relationship between unrelieved pain and cost of medical care, time spent in an intensive care unit, and length of hospital stay ${ }^{8}$.

Traditionally, postoperative pain has been managed either reactively with drugs given as needed or proactively with continuous infusion of analgesics ${ }^{9}$. Evidence suggests that reactive pain management is ineffective. With a reactive approach, analgesics are administered at the discretion of nurses and only on an as-needed basis. Consequently, treatment takes place after pain occurs ${ }^{10}$, causing some patients to experience severe pain ${ }^{11}$ In contrast, proactive pain management improves effectiveness, because treatment is given before pain occurs $^{10}$. With a proactive approach, analgesics are often administered via a continuous peripheral or epidural infusion. However, because this approach may entail added risk $^{5}$, is short-term, high-tech, equipment dependent, and often self-administered, it is neither available nor appropriate for all surgical patients ${ }^{12}$.

\section{Methods:}

The present study was contemplated to compare the efficacy between opioid and NSAID in the poststernotomy pain management following off-pump coronary bypass graft surgery, to see the influence of analgesics on haemodynamic state of the patients following off-pump coronary artery bypass graft surgery, to find out modulation of both early and delayed sequel of pain and to observe the restoration of respiratory function.

The present study was a prospective study which was carried out at Anesthesiology Department (cardiac anesthesia wing) of Bangabandhu Sheikh Mujib Medical University, Dhaka, over a period of 6 months from January 2009 to June 2009.

The study population was the patients scheduled for elective cardiac surgery and underwent off pump CABG with median sternotomy. Patients of ASA Grade I \& II between $40-60$ years were included in the study. Patients, who were not willing to participate in the study; had known contraindication to opioid or NSAID, impaired kidney \& liver function and previous operation with median sternotomy were excluded from the study. The demographic variables studied were age, sex and BMI. The safety variables were pulse rate, intra-arterial blood pressure (systolic and diastolic), respiratory rate, $\mathrm{SPO}_{2}$ and the outcome variable was intensity of post-sternotomy pain on visual analogue scale (VAS).
After selecting the patients based on selection criteria, they were divided into two study groups using random allocation procedure in Card lottery method. 30 patients were divided into diclofenac (Gr. A) and opioid (Gr. B)group - each containing 15 patients.

The patients of Group-A received diclofenac sodium 1 - 2 $\mathrm{mg} / \mathrm{kg}$ body weight 12 hourly in intramuscular route up to 48 hours, while the patients of Group-B received pethidine $1.5 \mathrm{mg} / \mathrm{kg}$ body weight 6 hourly in the same route as diclofenac sodium up to the same period. Observations were made at 6 hourly intervals up to 48 hours and the findings were compared between the groups to come to a decision which group was better in terms of outcome variable, postoperative intensity of pain.

Assessment of pain:

The uni-dimensional pain scales that can measure pain intensity and are self reported by patients are numerical rating scale, Verbal Rating Scale (VRS) and Visual Analogue Scale (VAS). Visual Analogue Scale (VAS) involves asking the patients to rate their pain from $0-10$ (11 points) with the understanding that 0 represents one end of pain intensity continuum (no pain) and 10 represents the other extreme of pain intensity (Unbearable pain). The strength of VAS is its simplicity and therefore can be used with a great variety of patients. Data were collected using a structured questionnaire (research instrument) addressing all the variables of interest.

Statistical analysis:

The test statistics used to analyze the data were descriptive statistics, Chi-square $\left(\chi^{2}\right)$ probability Test and repeated measure ANOVA. The level of significance was set at 0.05 and $\mathrm{p}<0.05$ was considered significant.

\section{Results:}

A total of 30 patients scheduled for elective cardiac surgery by off pump CABG with median sternotomy were planned to be treated by NSAID (Group-A) and opioid (Group-B) for postoperative pain management. Changes in pulse, systolic and diastolic blood pressures, respiratory rate, SPO2 and pain measured on VAS scale of the two groups were compared at 6 hourly intervals after extubation.

Data analysis demonstrated that no significant difference was found between groups with respect to Age and sex distribution $(\mathrm{p}=0.241)$.

The mean pulse rates of Group-A and Group-B were 108/ minute and 107/minute respectively at 6 hours of extubation which decreased to 96/minute and 103/minute respectively at 12 hours interval. Then both groups experienced a sharp 
rise and sharp fall with decrease of pulse rates to 94 and 99/minute at 24 hours interval. Thereafter the variable began to decrease insidiously and stabilized to 85 and 86/ minute in Group-A and Group-B respectively at the end of 48 hours. The changes in pulse rates were similar in both groups that means there is no significant difference $(\mathrm{p}=$ 0.836).

Fig..-1 depicts the changes in systolic blood pressure (SBP) at different time interval. At 6 hours of extubation, the mean SBPs of Group-A and Group-B were 149 and 132 mmHg respectively and continued decreasing up to 24 hours in Group-A and up to 30 hours in Group-B. Finally the blood pressures stabilized to 115 and $113 \mathrm{mmHg}$ in Group-A and in Group-B respectively with no significant difference between the groups ( $p=0.171)$.

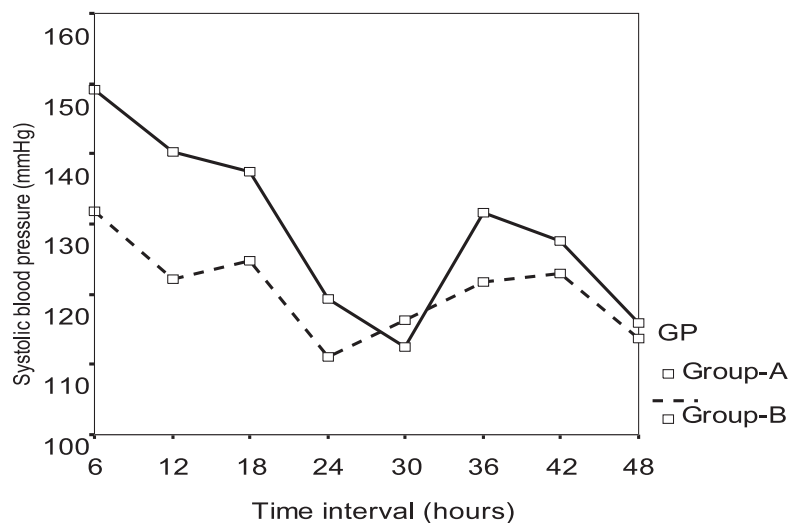

Fig.-1: Monitoring of SBP at different time interval

The mean diastolic blood pressure from 6 to 48 hours of observation were significantly

higher in Group-A than those in Group-B ( $p=0.021)$. (Fig.-2).

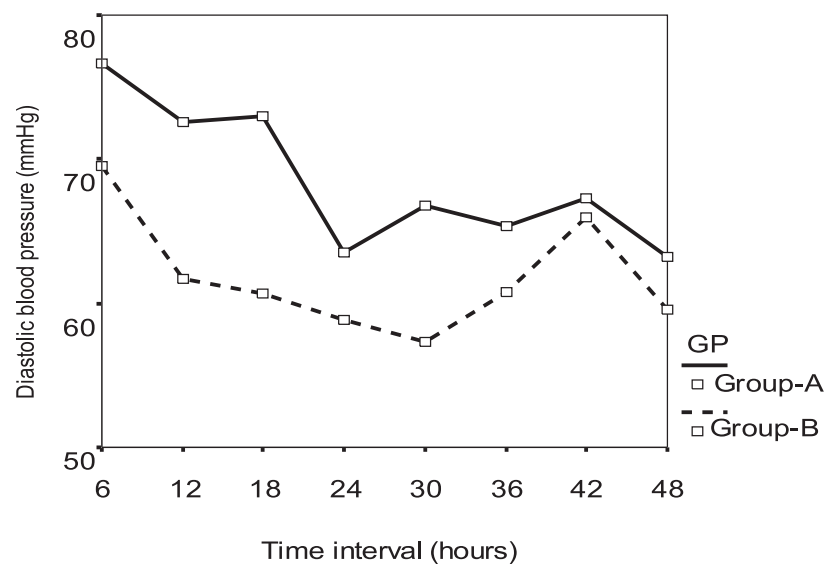

Fig.-2: Monitoring of Diastolic BP at different time interval
Fig.-3 explains the changes of respiratory rate at different time intervals. The rate was 19/minute in Group-A and 20/ minute in Group-B at 6 hours of extubation. Both groups had some ups and downs and finally stabilized to $17 /$ minute in either group $(\mathrm{p}=0.911)$.



Fig.-3. Monitoring of respiratory rate at different time interval

\section{Visual Analog Scale (VAS):}

The intensity of pain measured by visual analog scale (VAS) shows that the mean pain score of Group-A and Group-B were 6.9 and 6.8 respectively at 6 hours of extubation which decreased insidiously to 4.1 and 3.3 at 48 hours. The intensity of pain was significantly higher in the former group than that in the latter group throughout the whole period of observation ( $p=0.045$ ) (Table VIII \& Fig. 7).

Table-I

Pain score in VAS scale at different time interval

\begin{tabular}{lccc}
\hline Pain on VAS & Group A & Group B & $\begin{array}{c}\text { P- } \\
(0-10 \mathrm{~cm}) \text { at }\end{array}$ \\
\hline 6 hours & $6.9 \pm 0.4$ & $6.8 \pm 0.4$ & $0.63 \mathrm{NS}$ \\
12 hours & $6.3 \pm 0.4$ & $6.1 \pm 0.5$ & $0.31 \mathrm{NS}$ \\
18 hours & $5.7 \pm 0.5$ & $5.9 \pm 0.5$ & $0.134 \mathrm{NS}$ \\
24 hours & $5.5 \pm 0.6$ & $5.3 \pm 0.6$ & $0.045 \mathrm{NS}$ \\
30 hours & $5.3 \pm 0.9$ & $4.7 \pm 0.6$ & $0.080 \mathrm{Ns}$ \\
36 hours & $4.9 \pm 0.7$ & $4.5 \pm 0.7$ & $0.088 \mathrm{NS}$ \\
42 hours & $4.3 \pm 0.8$ & $3.5 \pm 0.5$ & $0.004 \mathrm{~S}$ \\
48 hours & $4.1 \pm 0.8$ & $3.3 \pm 0.4$ & $0.004 \mathrm{~S}$ \\
\hline
\end{tabular}

\# Repeated measure ANOVA statistics was employed to analyze the data and 'p' refers to overall differences between groups; $\mathrm{S}$ = Significant; NS = Not significant. 


\section{Discussion:}

Adequacy of postoperative pain control is one of the most important factors in determining when a patient can be safely discharged from a surgical facility and has a major influence on the patient's ability to continue their normal activities of daily living. ${ }^{15}$ Perioperative analgesia has traditionally been provided by opioid analgesics viz morphine, fentanyl etc. However, extensive use of opioids is associated with a variety of perioperative side effects, such as ventilatory depression, drowsiness and sedation, postoperative nausea and vomiting (PONV), pruritus, urinary retention, ileus, and constipation that can delay hospital discharge ${ }^{14,17}$. Although these side-effects are not encountered by the opioid group of patients in the present study as the selection of postoperative opioid is different here (pethidine), we must keep in mind the dangerous side effects of these drugs when planning for postoperative analgesia in individual patients with opioid group of drugs. Universally, the goal of peri-operative pain management is to provide a good outcome of surgery. The World Health Organization (WHO) has addressed this goal by developing a framework for managing pain. Accreditation standards have evolved and now include specific expectations about the management of pain ${ }^{13}$.

Intra-operative use of large bolus doses or continuous infusions of potent opioid analgesics may actually increase postoperative pain as a result of their rapid elimination and/or the development of acute tolerance ${ }^{16}$. In addition; it has been suggested by the Joint Commission on Accreditation of Healthcare Organizations that excessive use of postoperative opioid analgesics leads to decreased patient satisfaction. Partial opioid agonists (e.g., tramadol) are also associated with increased side effects (e.g., nausea, vomiting, ileus) and patient dissatisfaction compared with both opioid ${ }^{17}$ and non-opioid ${ }^{18}$ analgesics.

Some study reports earlier suggested that parenteral NSAID possessed analgesic properties comparable to the traditional opioid analgesics ${ }^{19}$ without producing any opioid-related side effects ${ }^{20}$. Compared with the partial opioid agonist tramadol, diclofenac produced better postoperative pain relief with fewer side effects after cardiac surgery $^{21}$. When administered as an adjuvant during outpatient anesthesia, diclofenac sodium was associated with improved postoperative analgesia and patient comfort compared with fentanyl and the partial opioid agonist, dezocine ${ }^{22}$. Other investigators reported that diclofenac sodium provided postoperative pain relief similar to that of fentanyl but was associated with less nausea and somnolence, as well as an earlier return of bowel function ${ }^{23}$. In most studies, use of diclofenac sodium has been associated with a less frequent incidence of PONV than the opioid analgesics. As a result, patients tolerate oral fluids and are fit for discharge earlier than those receiving only opioid analgesics during the perioperative period. Of interest, diclofenac sodium was superior to a dilute local anesthetic infusion (bupivacaine $0.125 \%$ ) in supplementing epidural PCA hydromorphone in patients undergoing thoracotomy procedures ${ }^{24}$. Furthermore, it has been found that the diclofenac sodium at the incision site in combination with local anesthesia resulted in significantly less postoperative pain, a better quality of recovery, and earlier discharge compared with local anesthesia alone ${ }^{24}$. In fact, there is evidence for both a peripheral and central analgesic action of NSAID. ${ }^{25}$ However, when diclofenac sodium was substituted for or combined with fentanyl during minor gynecologic and laparoscopic procedures; the beneficial effects of the NSAID were reduced ${ }^{26}$.

Despite the obvious benefits of using NSAIDs in the perioperative period, controversy still exists regarding their use because of the potential for gastrointestinal mucosal damage and renal tubular and platelet dysfunction ${ }^{27}$. Although some studies have found increased blood loss and risk of reoperation when diclofenac sodium was administered to children undergoing tonsillectomy procedures $^{28}$.

Opioid analgesics are a broad group of compounds that includes naturally occurring extracts of opium, synthetic surrogates, and endogenous peptides. Opioid receptors are widely distributed, and close voltage-dependent calcium channels, and opens calcium-dependent inwardly rectifying potassium channels, resulting in inhibitory effects characterized by neuronal hyperpolarization and decreased excitability ${ }^{29}$. Opioids are commonly administered throughout the perioperative period for cardiothoracic procedures. Intra-operatively, they are given intravenously as either the primary anesthetic agent or, more commonly, as an adjunct to a mixed anesthetic technique that includes potent inhaled anesthetics, benzodiazepines, and other agents. A primary benefit of effective pain control is patient satisfaction. Outcome benefits that involve peri-operative complications appear to be highly related to the analgesia technique used, particularly in relation to the effectiveness in blocking the surgical stress response and nociceptive spinal reflexes. Pavlin et al. confirmed that moderate-to-severe pain prolonged recovery room stay by $40-80 \mathrm{~min}^{30}$. Use of local anesthetics and NSAIDs decreased pain scores and facilitated an earlier discharge home. Additional outcome 
studies are needed to validate the beneficial effect of these non-opioid therapeutic approaches with respect to important recovery variables (e.g., resumption of normal activities, dietary intake, bowel function, return to work). Although many factors other than pain must be controlled to minimize postoperative morbidity and facilitate the recovery process ${ }^{8}$. Pain remains a major concern of all patients undergoing elective surgical procedures.

Opioid analgesics continue to play an important role in the management of moderate-to-severe pain after surgical procedures. However, adjunctive use of non-opioid analgesics will likely assume a greater role as minimally invasive ("key hole") surgery continues to expand ${ }^{31}$. In introducing new therapeutic modalities for pain management, it is important to carefully consider the risk: benefit ratio ${ }^{32}$. Use of local anesthetics and NSAIDs decreased pain scores and facilitated an earlier discharge home. In the present study, Diclofenac sodium and Pethidine are compared to find a better option, because pethidine is not a common choice for post-operative analgesia in cardiac surgery. But the visual analogue scale found the significance of difference in pain quality between them.

The optimal non-opioid analgesic technique for postoperative pain management would not only reduce pain scores and enhance patient satisfaction but also facilitate earlier mobilization and rehabilitation by reducing pain-related complications after surgery. The visual analog scale (VAS) has been used to assess the efficacy of pain management regimens in patients with acute postoperative pain. In this study, the intensity of pain measured on visual analog scale (VAS $0-10 \mathrm{~cm}$ ) demonstrated that the mean pain intensity of Group-A and Group-B were 6.9 and 6.8 respectively at 6 hours of extubation which decreased gradually to 4.1 and 3.3 at 48 hours. The intensity of pain was significantly higher in the NSAID group than that in the opioid (pethidine) group throughout the whole period of observation ( $p=0.045)$ indicating that use of Pethidine in the post-sternotomy pain management is better and faster than that of NSAID (diclofenac).previous pain experience, anxiety, or anticipated pain with consistency in VAS scores.

\section{Conclusion:}

From the findings of the study and discussion thereof it could be concluded that the intensity of post-sternotomy pain was significantly higher in the NSAID group than that in the opioid group throughout the whole period of observation suggesting that opioid (pethidine) though uncommon in cardiac surgery, would be a promising analgesic in the post-sternotomy pain management than that of NSAID (diclofenac sodium).It again proves the superiority of opioids as post-operative analgesics over NSAIDs, though many studies elaborated in discussion proved the efficiency and preference for it.

However, our study findings are based on small sample size which lacks generalization. Further study with large sample is, therefore, needed to arrive at a definitive conclusion. Moreover, the study did not consider the sideeffects encountered by the patients of two study groups. Risk benefit ratio of the two study drugs must be weighed before making a general recommendation as to which of the two drugs should be used in the management of poststernotomy pain following off-pump CABG.

\section{References:}

1. Adam pick 2008. The patient's guide to Heart valve surgery from http:/www. Heart valve surgery.com/sternum-brokenpain-healing.php.

2. Rahman AKMS. Management of post CABG pain; Bangladesh context, $5^{\text {th }}$ south Asian confederation of Anesthesia 2003:302-6.

3. Bainbridge D \& Martin JE. NSID - analgesia, pain control and morbidity in cardiothoracic surgery. Canadian Journal of Anesthesia 2006;53:46-59.

4. Aarhus University Hospital, December 2007 from http:/ clinical trials,gov/ct2 show/NCT 00572208.

5. Swarm RA, Karanikolas M, Kalauokalani D. Pain treatment in the perioperative period. Cur Probl Surg. 2001;38:835920.

6. Dracup K, Bryan-Brown C. Pain in the ICU: fact or fiction? Am J Crit Care.1995;4:337-9.

7. Watt-Watson J, Stevens B. Managing pain after coronary artery bypass surgery. J Cardiovasc Nurs. 1998;12:39-51.

8. Kehlet H. Acute pain control and accelerated postoperative surgical recovery. Surg Clin North Am. 1999;79:431-43.

9. Kruger M, McRae K. Pain management in cardiothoracic practice. Surg ClinNorth Am. 1999;79:387-400.

10. Melzack R. The tragedy of needless pain. Sci Am. 1990;262:27-33.

11. Carroll KC, Atkins PJ, Herold GR, et al. Pain assessment and management in critically ill postoperative and trauma patients: a multisite study. Am J CritCare. 1999;8:105-17.

12. Pasero C, McCaffery M. Multimodal balanced analgesia in the critically ill. Crit Care Nurs Clin North Am. 2001;13:195206.

13. Cancer Pain Relief and Palliative Care: Report of a WHO Expert Committee. Geneva, Switzerland: World Health Organization; 1990:1-75. World Health Organization Technical Report Series, 804. 
14. White PF. Ambulatory anesthesia advances into the new millennium. Anesth Analg 2000;90:1234-5.

15. Chung F, Ritchie E, Su J. Postoperative pain in ambulatory surgery. Anesth Analg 1997;85:808-16.

16. Guignard B, Bossard AE, Coste C, et al. Acute opioid tolerance: intraoperative remifentanil increases postoperative pain and morphine requirement. Anesthesiology 2000;93:409-17.

17. Silvasti M, Svartling N, Pitkanen M, Rosenberg PH. Comparison of intravenous patient-controlled analgesia with tramadol versus morphine after microvascular breast reconstruction. Eur J Anaesthesiol 2000;17:448-55.

18. Rawal N, Allvin R, Amilon A, et al. Postoperative analgesia at home after ambulatory hand surgery: a controlled comparison of tramadol, metamizol, and paracetamol. Anesth Analg 2001;92:347-51

19. Yee JP, Koshiver JE, Allbon C, Brown CR. Comparison of intramuscular ketorolac tromethamine and morphine sulphate for analgesia of pain after major surgery. Pharmacotherapy 1986;6:253-61.

20. Ding Y, White PF. Comparative effects of ketorolac, dezocine and fentanyl as adjuvants during outpatient anesthesia. Anesth Analg 1992;75:566-71.

21. Immer FF, Immer-Bansi AS, Tachesel N, et al. Pain treatment with a COX-2 inhibitor after coronary artery bypass operation: a randomized trial. Ann Thorac Surg 2003;75:490-5.

22. Ramirez-Ruiz M, Smith I, White PF. Use of analgesics during propofol sedation: a comparison of ketorolac, dezocine, and fentanyl. J Clin Anesth 1995;7:481-5.

23. Wong HY, Carpenter RL, Kopacz DJ, et al. A randomized double-blind evaluation of ketorolac tromethamine for postoperative analgesia in ambulatory surgery patients. Anesthesiology 1993;78:6-14.

24. Coloma M, White PF, Huber PJ, et al. The effect of ketorolac on recovery after anorectal surgery: IV versus local administration. Anesth Analg 2000;90:1107-10.

25. Romsing J, Moiniche S, Ostergaard D \& Dahl JB. Local infiltration with NSAIDs for postoperative analgesia: evidence for a peripheral analgesic action. Acta Anaesthesiol Scand 2000;44:672-83.

26. Liu J, Ding Y, White PF, et al. Effects of ketorolac on postoperative analgesia and ventilatory function after laparoscopic cholecystectomy. Anesth Analg 1993;76:10616.

27. Souter A, Fredman B, White PF. Controversies in the perioperative use of nonsteroidal antiinflammatory drugs. Anesth Analg 1994;79:1178-90.

28. Gunter JB, Varughese AM, Harrington JF, et al. Recovery and complications after tonsillectomy in children: a comparison of ketorolac and morphine. Anesth Analg 1995;81:1136-41.

29. Bovill JG. Update on opioid and analgesic pharmacology.Anesth Analg2001; 92:S1-S5.

30. Pavlin DJ, Chen C, Penaloza DA, et al. Pain as a factor complicating recovery and discharge after ambulatory surgery. Anesth Analg 2003;97:1627-32.

31. White PF. The role of non-opioid analgesic techniques in the management of pain after ambulatory surgery. Anesth Analg 2002;94:577-85.

32. White PF. Changing role of COX-2 inhibitors in the perioperative period: is parecoxib really the answer? Anesth Analg 2005;100:1306-8. 